Sinchiz-Lafuintt, J., 1986: La epigrafia y el entorno arqueológico de la villa romana de Gárgoles de Arriba (Guadalajara), Lucentum, V, págs. 175-182, Alicante.

SAnchez Rfal, J., 1951: Los mosaicos romanos de Pared Delgada, Boletín Arqueológico, 34, págs. 108-109, Tarragona.
SANz, R., 1987: Mosaicos romanos del Camino Viejo de las Sepulturas (Balazote, Albacete), Al-Basit, 21, págs. 43-64, Albacete.

TARACENA, B. 1946: El palacio romano de Clunia, Archivo Español de Arqueologia, XIX, págs. 29-69, Madrid.

\title{
INSCRIPCIONES MUSIVAS EN LA ANTIGÜEDAD TARDÍA HISPANA
}

POR

\author{
JOAN GÓMEZ PALLARÉS
}

Universitat Autónoma de Barcelona

\section{RESUMEN}

Este trabajo revisa y corrige las páginas dedicadas a las inscripciones musivas de Hispania en el libro de M.Guardia, Los mosaicos de la Antigüedad lardia en Hispania. Estudios de iconografia, Barcelona, 1992.

\section{SUMMARY}

This paper tries to revise and correct the pages devoted to Hispania's mosaic inscriptions, contained in M.Guardia's Los mosaicos de la Antigüedad tardia en Hispania. Estudios de iconografia. Barcelona, 1992.

\section{INTRODUCCIÓN}

En el año 1988, la Profesora Milagros Guardia defendía su Tesis Doctoral sobre el tema Temática y programas iconográficos en la musivaria hispano-romana del Bajo Imperio' y hoy nos llega la publicación definitiva de ese trabajo, con el título de Los mosaicos de la Antigüedad tardia en Hispania. Estudios de iconografia ${ }^{2}$. A lo largo de estas páginas quisié-

1 Tesis Doctoral publicada por la Universidad de Barcelona, en su colección de microfichas Tesis Doctorales Microfichadas, n.488, Barcelona, 1989 (ISBN 84-7528-706-9).

2 Publicado por las Promociones y Publicaciones Universitarias, S.A., en Barcelona, 1992 (ISBN 84-7665944-X). ramos presentar algunas observaciones a esta publicación, centrándonos en exclusiva en aquello en que podemos decir algo sólido, es decir, en las inscripciones musivas contenidas en los pavimentos estudiados por la Dra.Guardia ${ }^{3}$. Estas observaciones se van a fijar en el «Apéndice B» del libro de referencia (págs. 400-412) donde se recogen «las inscriciones que presentan el conjunto de los mosaicos hispánicos del Bajo Imperio». Si es necesario, también nos referiremos a las páginas precedentes donde las inscripciones se estudian en su ambiente arqueológico y edilicio ${ }^{4}$.

${ }^{3}$ En el momento en que escribimos estas páginas (mayo de 1993) se encuentra en curso de publicación nuestro Corpus de Inscripciones Musivas de Hispania (CIMH), que constará de dos secciones diferenciadas. Un primer volumen, que será publicado por la Academia Española de Historia y Arqueología de Roma, se centra en las inscripciones paganas, mientras que una segunda entrega (en formato articulo), trata las inscripciones cristianas.

4 Algunas de estas inscripciones son presentadas en el ámbito de estudios iconográficos sobre los pavimentos figurados de Hispania, que forman la parte importante de este trabajo (págs. 29-293) y que valoro mucho, pero sobre lo que, en conciencia, no puedo hablar: aquello que decia Cicerón, non possumus omnes adire $\mathrm{Co}$ rinthium. Las inscripciones son citadas también en el "Apéndice A" (págs. 373-399), donde se recoge "el conjunto de viviendas con mosaicos figurados bajo-imperiales que no han sido seleccionados en el estudio previo (pág. 373)m. 
En el año 1989, cuando empezaba a estudiar las inscripciones musivas de Hispania, hice una primera lectura de la Tesis de la Dra.Guardia, gracias a un permiso escrito suyo, que siempre he agradecido. Sus páginas fueron para mi de gran ayuda en esos momentos. Casi cinco años después de esa primera lectura, recibí con alegria y esperanza la noticia de la publicación definitiva de ese trabajo pensando que su información y la mía habrian crecido al mismo tiempo y su integración favoreceria a ambas. Pero una lectura atenta de la información que más me interesaba del libro da la Dra.Guardia me demuestra que no ha sido así.

Las páginas de 1992 han salido a la luz sin grandes cambios y ahi radica el problema. No es lo mismo leer en 1989, en una Tesis inédita, que «no pretendemos realizar una minuciosa edición de estas inscripciones", que encontrar exactamente la misma afirmación en la publicación definitiva y divulgada de 1992.

Soy consciente de que la especialidad de la Dra.Guardia no es la epigrafia, como no es la mía la iconografia en los mosaicos hispanos. Non omnia possumus omnes nos aconseja Virgilio nec scire fas est omnia recomienda su amigo Horacio. La reproducción en 1992 de las páginas de 1988 nos ha llevado a escribir estas notas para corregir algunas de las ediciones que se leen en el libro de la Dra.Guardia porque, aunque no se trate de "una minuciosa edición», en ese libro se presentan las inscripciones de los mosaicos figurados hispanos; porque, aunque no se trate de «una minuciosa edición», la autora "acepta lecturas existentes o propone otras" (mezclando, por tanto, lecturas propias con otras procedentes de fuentes diversas) y porque, aunque no se trate de "una minuciosa edición", "transcribe las inscripciones según las normas de la reedición actualizada del $C I L \mathrm{II}^{5}$ ").

El orden de mis notas sigue el de la presentación de las inscripciones en el libro de la Dra.Guardia.

1. En la edición del llamado «Mosaico del Circo de Barcelona ${ }^{6}$, , la Dra.Guardia no ofre-

\footnotetext{
3 Todas estas afirmaciones se encuentran en la pág. 400.

- Para este mosaico se puede ver CIL II 5129 y 6146 ; IRB 119; ILER 2108; Piernavieja 1977, n.21; Barral 1978.
}

ce (pág. 401) el texto de la inscripción de lo que habitualmente se ha considerado un obelisco encima de la spinna y que nosotros, en cambio, preferimos identificar con un nilómetro. Aunque no podamos descifrar con exactitud el contenido de la inscripción, queda al menos claro que ahi existian letras o símbolos (para nosotros, números), en alfabeto griego,

N.6 y CIMHI, n.B 4. Además de CIMH I, las abreviaturas utilizadas en este trabajo son las siguientes:

- Alarcâo 1988 = J.Alarcâo, Roman Portugal. Wardminster, 1988.

- Álvarez 1990 = J.M. Álvarez Martinez, Mosaicos Romanos de Mérida. Nuevos hallazgos. Monografias Emeritenses, 4. Madrid. 1990.

- Balil $1965=$ A.Balil, "Algunos mosaicos romanos de època tardian, Principe de Viana, 100-101 (1965), págs. 281-293.

- Barral 1978 = X.Barral, Les mosuiques romaines et médievales de la Regio Laietana (Barcelona el ses environs). Barcelona, 1978

- Blázquez-González Navarrete 1974 = J.M.BlázquezJ.González Navarrete, «Mosaicos hispánicos del Bajo Imperiom, AEArq, $45-47$ (1972-74), págs. 429-432.

- BSAA = Boletin del Seminario de Arte y Arqueologia. Univ. de Valladolid.

- $C I L=$ Corpus Inscriptionum Latinarum. CIL II = Inscriptiones Hispaniae Latinae. Indices. Edidit E. Hübner. Berlin, 1869 y Inscriptiones Hispaniae Antiquae Supplementum. Indices, Berlin, 1892.

- Corpus Mosaicos I = Corpus de Mosaicos Romanos de España. I. Mosaicos Romanos de Mérida. Por A.Blanco Freijeiro, Madrid, 1978.

* Corpus Mosaicos III = Corpus de Mosaicos Romanos de España. III. Mosaicos Romanos de Córdoba. Jaén y Málaga, Por J.M.Blázquez, Madrid, 1981.

- Corpus Mosaicos V = Corpus de Mosaicos Romanos de España. V. Mosaicos Romanos de la Real Academia de la Historia, Ciudad Real, Toledo, Madrid y Cuenca. Por J.M.Blázquez, Madrid, 1982.

- Corpus Mosaicos VIII = Corpus de Mosaicos Romanos de España. VIII. Mosaicos Romanos de Lérida y Albacete. Por J.M.Blázquez, G. López Monteagudo, M.L. Neira y M.P.San Nicolás Pedraz, Madrid, 1989.

- Corpus Mosaicos IX = Corpus de Mosaicos Romanos de España. IX. Mosaicos Romanos del Museo Arqueológico Nacional. Por J.M.Blázquez, G.López Monteagudo, M.L.Neira y M.P.San Nicolás Pedraz, Madrid, 1989.

- Chaves $1956=$ L.Chaves, «Estudos lusitano-romanos. 1. A Villa de Santa Vitoria do Ameixial. Escavaçoes em 1915-1916", AP, 30 (1956), págs. 14-117.

- Donderer $1989=$ M.Donderer, Die Mosaizisten der Antike und ihre wirtschaftliche und soziale Stellung. Eine Quellenstudie, Erlangen, 1989.

- Fernández-Galiano 1984 = D.Fernández-Galiano, Complutum II. Los mosaicos, EAE 138, Madrid, 1984.

- Fernández-Galiano 1987 = D.Fernández-Galiano, Mosaicos Romanos del convento cesaraugustano, Zaragoza, 1987. 
que tienen que ser editados o comentados cuando se habla de esta inscripción.

Nuestra edición:
I I I
I I I
ता $\mathrm{T}$
$\Delta \Gamma \mathrm{O}$
$\mathrm{MZ}$
$\Theta \Delta$

* Fernandez-Galiano 1989 = D. Fernández-Galiano, «La villa de Maternon, Mosaicos Romanos 1989, págs.255-269.

* Gómez Pallarès 1991 = J.Gómez Pallarès, "Nombres de artistas en inscripciones musivas latinas e ibéricas de Hispanian, Epigraphica 53 (1991), págs.59-96.

* Gómez Pallarés e.p. = J.Gómez Pallarès, «Varia Musiva Epigraphica, 1-VII", Conimbriga, 30 (1991), en prensa.

* Gorges $1979=$ J.-G.Gorges, Les villas hispano-romaines. Inventaire el problematique archeologiques, Paris. 1979 .

* HE I = Hispania Epigraphica I (1989). Ministerio de Cultura. Univ. Complutense. Madrid, 1989.

* ILER = J.Vives, Inscripciones Latinas de la España Romana. Antologia de 6.800) textos, Barcelona, 1971.

* IRB = S.Mariner Bigorra. Inscripciones Romanas de Barcelona (lapidarias y musivas). Barcelona, 1973.

* IRC II = G.Fabre-M.Mayer-I.Roda, Inscriptions Romaines de Catalogne. II. Lirida. Paris, 1985.

* IRC III = G.Fabre-M.Mayer-I.Rodà, Inscriptions Romuines de Catalogne. III. Gerone, Paris, 1991.

* Lancha 1984 = J.Lancha, "Les mosaïstes dans la vie économique de la Péninsule Ibèrique du I au IV siècle. Etat de la question et quelques hypothèses". MCV, 20 (1984), págs. 45-61.

* MCV = Mélanges de la Casa de Velázquez, Madrid.

* $\mathrm{MM}=$ Madrider Mitteilungen.

* Mosaicos Romanos 1989 = Mosaicos Romanos. In memoriam M.Fernández-Galiano. Actas de la I Mesa Redonda Hispano-francesa sobre Mosaicos Romanos, Madrid, 1989.

* Palol 1963 = P. de Palol, «El mosaico de tema oceánico de la villa de Dueñas (Palencia)", BSAA, 29 (1963). págs. 5-34.

* Piernavieja 1977 = P.Piernavieja, Corpus de inscripciones deportivas de la España Romana, Madrid, 1977.

* Rabanal-Abascal $1985=$ M.A.Rabanal-J.M.Abascal. "Inscripciones romanas de la provincia de Alicante", Lucentum, 4 (1985), págs. 191-245.

* Rodà e.p. = 1. Rodà, "Iconografia y epigrafia en dos mosaicos hispánicos: las villas de Tossa y de Dueñas», VI Coloquio Internacional del Mosaico Antiguo, Palencia-Mérida. 1990. en prensa. Queremos agradecer a la Prof.Rodà su amabilidad al dejarnos consultar su trabajo todavía inédito.

* Schlunk-Hauschild $1978=$ H.Schlunk-Th.Hauschild, Die Denkmäler der frühchristlichen und westgotischen Zeit, Mainz a. Rhein, 1978.

El único signo diacrítico que utilizamos en este trabajo y que no es habitual en los repertorios epigráficos es el del nexo, que indicamos con $/ \wedge /$ entre las dos letras unidas, v.g. $O F / F / C^{\wedge} N^{\wedge} A$, en nuestra observación al texto n.2 (vid. infra).
2. En el mosaico encontrado en la villa de Tossa de Mar (Girona) ?, existe un nexo entre $N$ y $A$ que la Dra.Guardia no indica en su edición (pág. 402).

Edición Guardia:

Salvo

Vitale felix Turissa (parte superior)

Ex. of

ficina Felices (parte inferior)

Edición nuestra:
a. SALVO
VITALE FELIX TVRISSA
b. EX. OF FICIN^A FELICES

3. En la edición del llamado «Mosaico del Circo de Bell-Lloch» (Girona) ${ }^{x}$, pero también en otros puntos, la Dra.Guardia (págs. 402-403) no respeta la compaginación original de la inscripción. Por ejemplo, donde en Guardia se lee Filoromus, debería leerse FILORO/MVS. Existe, además, un punto final en la inscripción que Guardia no indica.

Edición Guardia:

Limenius

Euplium

Calimorfus

Patinicus

Polystefanus

Torax

Filoromus

Pantaracus

Cecilianus. ficet

Edición nuestra:

\section{a.1. FILORO MVS \\ a.2. PANTARACVS}

' Cf. ILER 2105; Gorges 1979, n.GE 26; IRC III II y CIMHI, n.GI 6.

${ }^{*}$ Cf. CIL II 6180; ILER 2104; Donderer 1989, n.A 5I; IRC III 6 y CIMH I, n.GI 5 . 


\section{b.1. Torax \\ b.2. POLY[STEF] \\ ANVS \\ c.I. CALIMORFVS \\ c.2. PATINICVS \\ d.I. LIMINIVS; \\ d.2. EIPI.IVM}

\section{c. CFCII IANVS, FICI:T.}

4. In la zona $\mathrm{N}$ del implurimm de la villa de Albesa (Lérida), fue puesto al descubierto un mosaico en el que se representaba, en un ambiente de decoración floral, un cesto en el interior del cual figuraba una inscripción ${ }^{4}$. En este caso, la Dra.Guardia confia en la lectura de R.Pita (c) nota 9) y desconoce la corrección publicada por IRC II en el año 1985, tres años antes de la defensa de la Tesis y siete años antes de la publicación de 1992. La inscripción tiene que leerse empezando por la linea inferior e interpretarse como una conocida formula ${ }^{10}$ que desea al propietario una feliz utilización del pavimento y de su casa.

Edicion Guardia:

\section{Receli vie}

Edición IRC II $15(\mathrm{c}$. figura I):

\section{REFELI $[X]$ \\ VTE}

5. El mosaico cristiano procedente de Estada (Huesca) todavia no ha sido leido o explicado satisfactoriamente. Es un mosaico que presenta grandes dificultades textuales e iconográficas, muestras de las cuales son las múltiples interpretaciones y ediciones del texto ${ }^{1 !}$. La Dra.Guardia

liid. R.Pita, "Inscripción del mosaico B de la villa romana de Albesas, Ampurias, 30 (1968), pág. 333; F.Lara Peinado. Epigrafia Romana de Lerida, Lerida, 1973, n.26: IRC II I5; Corpus Mosaicos VIII. n.2 y HE: 4 I8.

i" Cf. CIMHI, n.BA I, b., FEI.IX (procedente de Emerita Augusta): TO 1.1.3, ITERE FELIX MATERNE (de Carranque) y POR 7. ITERE F(elix) (de Torre de Palma. Monforte).

Para nosotros la bibliografia mas interesante es: II.ER 2107; Balil 1965, p.284; Blázqués-Gonzales Navarrete 1974, pàgs 429-432; J.Lostal, Arqueologia del Aragoin Romano. Zaragoza, 1980, págs. 55-58; J.Pons Sala, «Al-

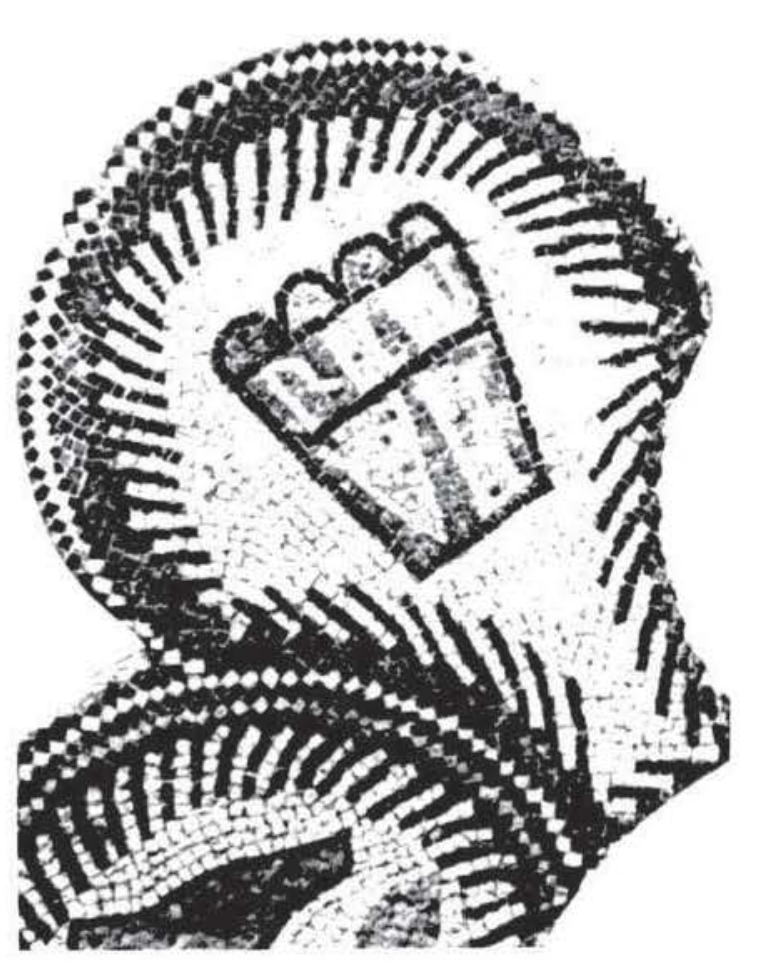

figura I Mosatico de lat cona $\mathrm{N}$ del tmplustum de la villa de sllesa (Lerida).

(pág. 403) sugiere una lectura que ucontempla algunas variantes con respecto a las propuestas...p, es decir, su libro ofrece una lectura propia y diferenciada de las otras, pero como ya sucede con ediciones precedentes, del texto propuesto por la Dra.Guardia se entienden bien pocas cosas.

\section{Edición Guardia:}

Dividimus muros et [moenia pandimus urbis] [-- ]o per singula +++

nescis quid [ter?] reret ossis veras cum A OUM (sic)

tibi condemnaca țantum repones poes

Hace dos años que el Prof. M. Mayer y yo mismo trabajamos con este texto, in situ y a

guns aspectes de la Ribagorça de l'época romana a través de l'epigrafia i la documentació visigotica i medievals. Ampurias, 45-46 (1983-84), págs, 230-231: F Beltran. "Epigrafia y Romanización en la provincia de Huesca». Annales. 4 (1987). p.30 y Furnandez-Galiano 1987, n.111. 
través de fotografias. La edicion del texto que hoy podemos proponer es esta (c\%. figura 2):

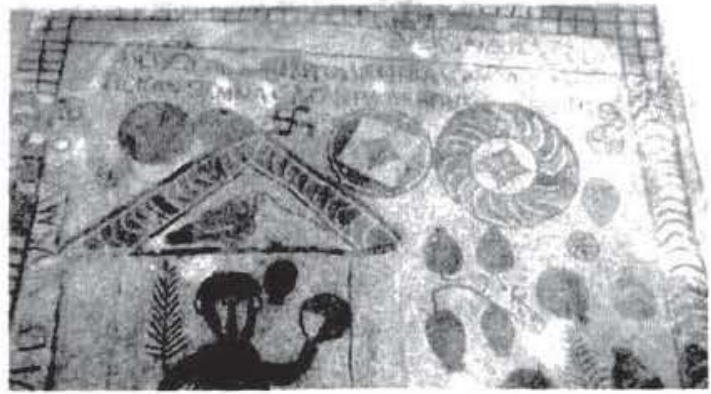

figura 2. Mosaleo eristiano prosedente de L tada (Huescal)

a. DIVIDIMVS MVROS ET [MOENIA PANDIMVS VRBIS?]

b. [-..]O PER SINGVLA GVS[TAS?]

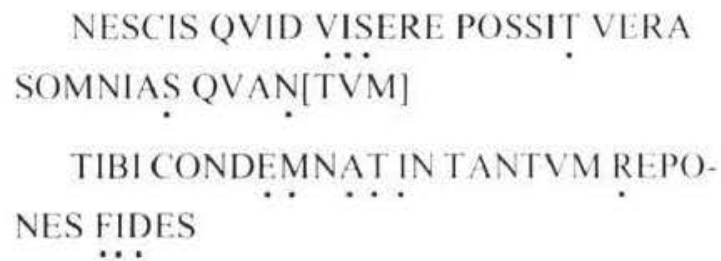

Las pausas sintácticas scrian: Dicidimus muros el /moenia pandimus urbis?] ${ }^{12}$. [-- - o per singula gus [tas]. Nescis quid visere possit. Vera somnias. Quan/tum] tibi condemnat, in tantum repones fides. El siguiente problema a solucionar es el de la relación entre texto e iconografia ${ }^{13}$, pero esa es una cuestión que no atañe a estas páginas.

6. De la llamada "Casa de Leda", en $\mathrm{Com}$ plutum (Alcalá de Henares, Madrid), procede un mosaico cuyo emblema central representa la seducción de Leda por parte de Zeus ${ }^{14}$. El mosaico nos da el título de la escena y nos identifica a la protagonista femenina de la misma.

\footnotetext{
12 Por el estado de conservación no podemos saber si el verso estaba completo o no en el mosaico. En cualquier caso, se trata de Verg., Aen. 2. 234

17 Estamos preparando un trabajo monográfico sobre el tema, que va a presentar todas las hipótesis de explicación del mosaico.

4 Vid. Fernández-Galiano 1984, págs. 203-213: HE । 463 y $C I M H I$, n.M 2.
}

La Dra. Guardia (pag. 405) ofrece una edicion del texto de la que parece deducirse que ha sido Leda la responsable del adulterio y no Zeus.

\section{Edicion Guardia:}

Adulterium Leda

lovis

Edicion nuestra (c\%. figura 3 ):
a. ADVLTLRIVMIOVIS
b. LFDA

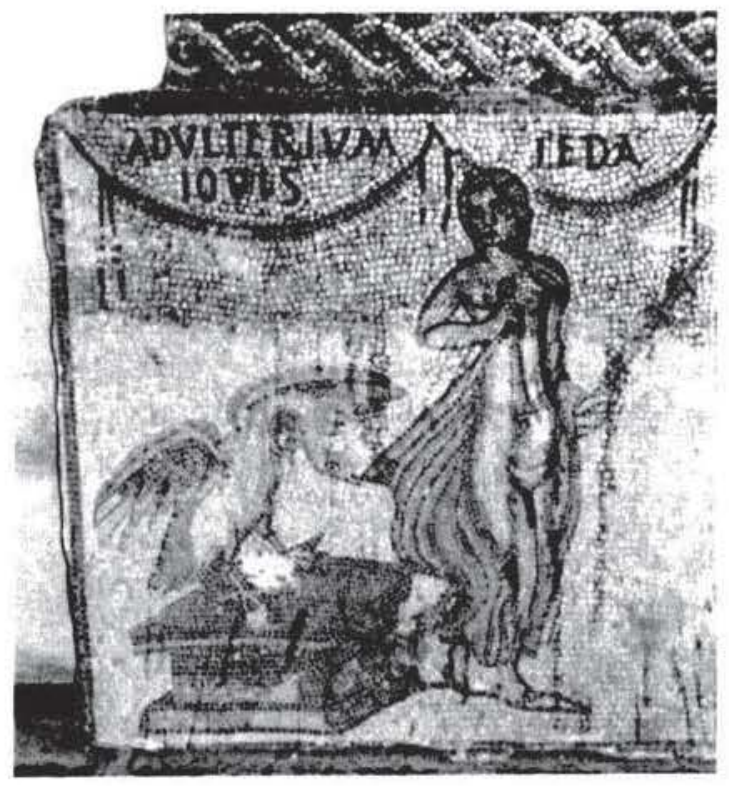

Figura 3.-Mosaico de la "easa de Leda”. (omphumm (Aleala de Henares, Madrid)

7. En la edición de una inscripción aislada procedente de un mosaico de la Alcudia de Elche (Alicante) ${ }^{15}$, en la que se identifica a la nereida protagonista del cuadro, la Dra.Guardia (pág. 405), no indica que existe una hédera cerrando el texto.

Edición Guardia:

Galatea

Edición nuestra:

GALATEA (hedera)

is Vid. CIL II 3554; ILER 2110; Rabanal-Abascal 1985. n.97; Corpus Mosaicos IX. n. 17 y CIMH I. n.A 2 
8. En la villa de Santisteban del Puerto (Jaén), salió a la luz uno de los pocos mosaicos de tema homérico de la Peninsula Ibérica ${ }^{\text {th }}$. En el se representa el pasaje de la lliada en que Aquiles es escondido por su madre Tetis en la isla de Skyros. entre las hijas de Licomedes. Alli. disfrazado convenientemente de muje y con el nombre de Pyrra, corteja a una de las hijas, Deidamia. En el mosaico se mezclan, a nuestro entender, otros temas del ciclo homerico, como son la presencia de Circe y de su sobrina Medea o el nombre de Priamo ${ }^{17}$. La edición de la Dra.Guardia (pág. 405) es una lectura propia, "que introduce algunas variantes" y que nos presenta el texto de una forma equivoca. De la compaginación que da a su edición (falsas separaciones entre las lineas y presentación de las distintas inscripciones como si se tratara de un solo texto), parece deducirse la existencia de alguna relación entre los personajes que. después, no se observa en el mosaico. Además, algunas de las lecturas consideradas por la Dra. Guardia como desaparecidas, pueden todavia hoy leerse en el mosaico.

Edición Guardia:

Pyrra / fili[us] / Tetidis / [C]irce Deidamia / Moedia. Iste

enim omne[s] virgines que sunt mu/lieres filiae sunt solis nam

[- - ] ] dis filius Priami.

Edición nuestra ( $c f$. figura 4):
a. MOEDIA
b. PY RRA
FILI VS
TETIDIS
c. [C]YRCE
d. DEIDAMIA

\author{
c. ISTE ENIM OMNES VIRGINES QVE \\ SVNT MV \\ LIERES FILIAE SVNT SOLIS \\ NAM - . - !! DIS FILIVS PRIAMI
}

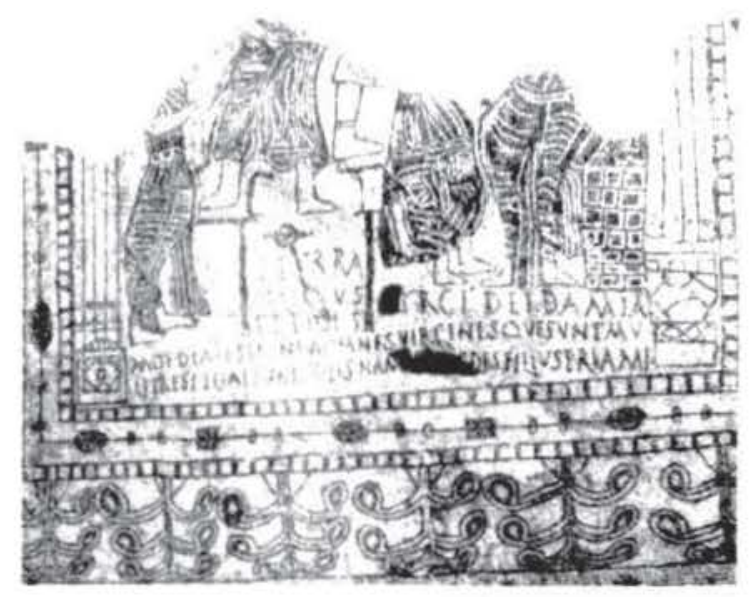

ligura 4. Iilla de Santiesteban del Puerto (Jaén) Mosaico de tema homérice.

9. Un pavimento procedente de una villa del pueblo de Saucedo (municipio de Talavera de la Reina, Toledo) ${ }^{1 k}$ presenta un emblema con un busto, a nuestro entender, masculino y una inscripción que identifica al personaje. La Dra.Guardia $(p .406)$ reproduce la lectura de Blázquez (Corpus Mosaicos V), pero expresa sus dudas sobre esa edición. Yo pienso que, sobre las fotos del mosaico (no se puede visitar por las obras que se están realizando en el palacio de Santa (ruz, donde se encuentra), no puede verificarse /seaius? (Blázquez y Guardia), sino ISCALLIS.

10. Procedentes de la villa romana de Carranque (Toledo), conocemos algunos pavimentos con decoración figurada e inscripción ${ }^{19}$. En el trabajo de la Dra.Guardia se encuentran en la pág. 406.

\footnotetext{
IN Vid. Corpus Mosaicos V. n.31: Gòmez Pallarès e.p. s.n. VI y CIMH I, n. TO 4

Vid., como primera introducción a la gran riqueza pavimental de esta villa. D.Fernández-Galiano-R.Garcia Serrano, "Carranque. Una villa con excepcionales mosaicos en el campo de Toledom. Historia 16, 152 (1988), págs. 105-115 y Fernández-Galiano 1989
} 
La primera es la perteneciente al cubiculum del dominus de la villa " y la lectur a de Guardia "introduce una variante" en la segunda linea: pincit por PINGIT. En nuestra opinión. e incluso si dejamos de lado ahora la cuestion de la regularidad del verbo, que nos llevaria a adoptar la forma pingit. no hay ninguna razon en el texto para leer ('en ves de $G$ : se puede comprobar en la fotografia que todas las ( $\mathrm{de}$ la inscripción tienen su apéndice inferior claramente dirigido hacia la parte superior de la caja de escritura: en cambio, la pretendida $C$ de la edición de la Dra.Guardia, en la forma pincit. muestra sin ningún tipo de dudas el apéndice inferior dirigido hacia la parte inferior de la caja. Asi pues, nuestra propuesta es PINGIT y no pincit.

Edición nuestra ( $c$ f. figura 5):
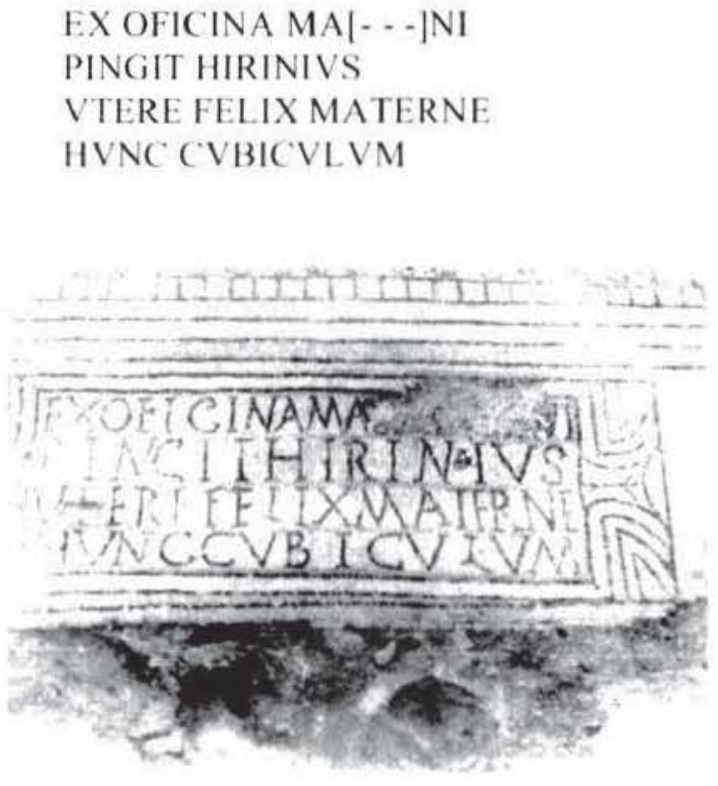

Figura 5. - Villa romana de (arranque (Toledo). Mosaico del cubiculum del domimus de la villa.

La segunda inscripción pertenece al triclinium de la villa ${ }^{21}$. La edición de la Dra.Guardia

25 Vid. J.Arce, "El mosaico de las Metamorfosis de Carranque (Toledo)m, MM, 27 (1986), págs. 365-374; Donderer 1989, n.A 68: Fernảndez-Galiano 1989, pảgs. 258259; HE I 607 y $C I M H$ I, n.TO 1.

21 Vid. Donderer 1989, n.A 64: Fernảndez-Galiano 1989 , p. 260; Gomez Pallarès 1991, n.1.7 y CIMH I, n.TO 3. no indica que en la tercera y cuarta palabras existe un espacio de texto perdido y, por tanto, no ofrece ningun intento de restitucion. Este último aspecto. sin duda, pertenece a la libre eleccion del investigador.

Edicion Guardia:

Ex officina iul prud (sic)

Edicion nuestra:

EX OFFICIN[A]IVL[II] PRVD[ENTIS?]

La tercera inscripción pertenece al oecus de la villa y contiene una representación de Marte y Venus, contemplando la lucha terrible entre Adonis y el jabali. Dos perros de caza, separados entre si e identificados por dos inscripciones, acompañan a Adonis "2. Si interpretáramos correctamente la edición de la Dra.Guardia, tendriamos que entender que se trata de una unica inscripción compuesta por dos lineas de texto, sin nexos de ningún tipo. La realidad es bien distinta.

\section{Edición Guardia:}

Leander/Titurus

Edición nuestra:

\section{a. LE.ANDER \\ b. TITVR^VS}

11. En el cercado de San Isidro (Dueñas, Palencia) fue puesta al descubierto una villa, en las termas de la cual (entre el caldarium y el tepidarium) salió un mosaico con representación de caballo y caballero a pie ${ }^{21}$. Una inscripción en el cuello del animal nos da su nombre. Quizá se trate de un error tipográfico, pero la edición de Guardia ofrece un texto Amori, mientras que en el texto se leia muy bien $A M O R / S^{24}$.

2 Vid. Fernảndez-Galiano 1989, págs. 259-260; D. Fernández-Galiano, "La villa de Materno. Carranque, Toledon, Revista de Arqueologia, 127 (1991), págs. $32-33$ y CIMHI, n.TO 2.

"Vid. Palol 1963; P. de Palol, "Das Okeanos-Mosaik in der rōmischen villa zu Dueñas (prov. Palencia)», MM. 8 (1967), págs, 196-225: Rodà e.p. y C $/ M H$ I. P I.

s4 Un problema de indole distinta es la consideración que meresca una probable $C$ junto al arnés del animal y cerca de la palabra Amoris. En opinión de Roda, compartida por nosotros, podria tratarse de una abreviatura de 

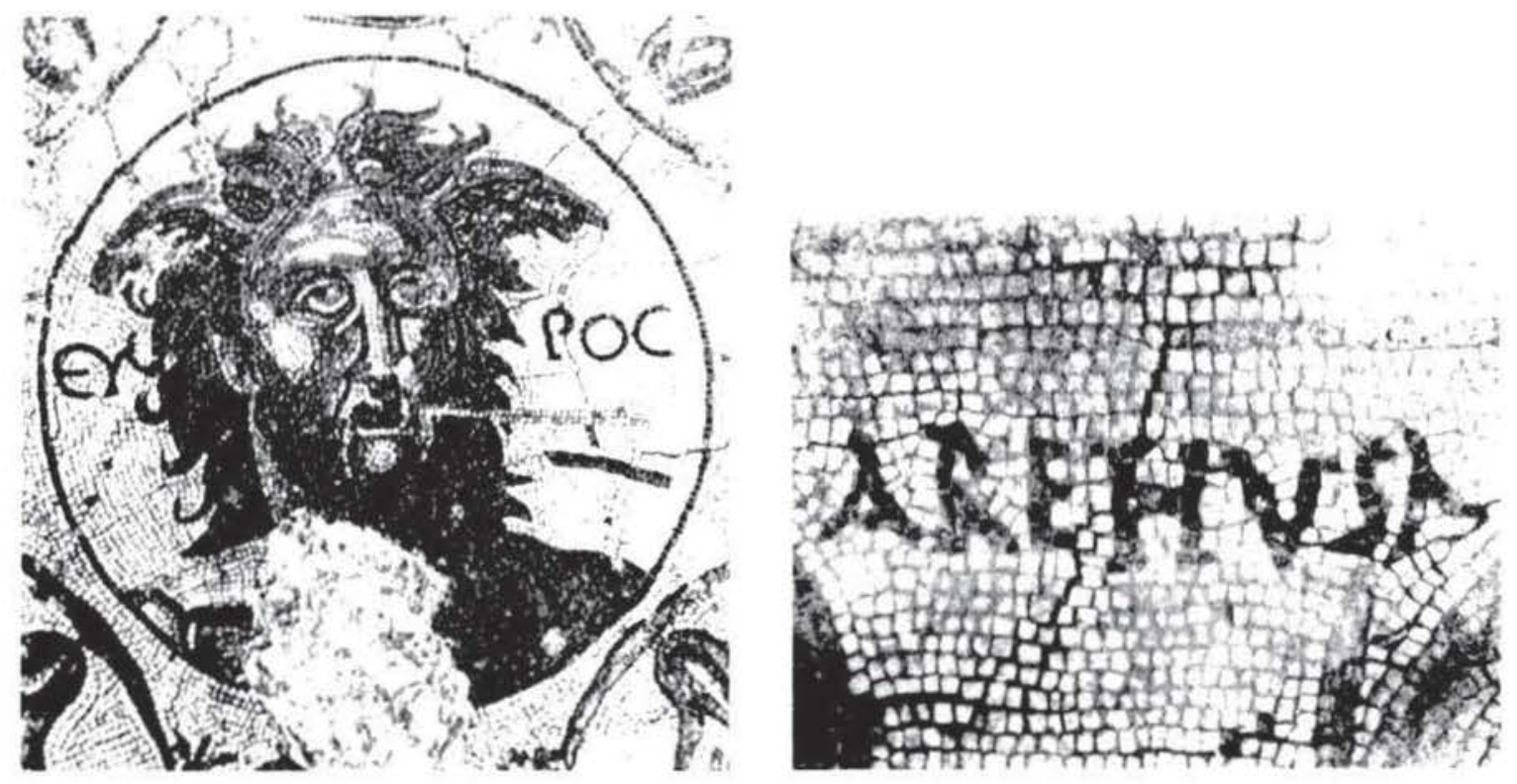

Figura 6. Mosaico de lfaliea. Nacimiento de Venus. Detalles de las imseriperenes

12. Tres $\mathrm{km}$ al sur del pueblo de Cabezón de Pisuerga (Valladolid) ${ }^{25}$, apareció otro mosaico de tema homérico, que combina imágenes y texto ${ }^{26}$. El mosaico representaba un pasaje de la lliada (II., 6. 119-236 y, más concretamente, 119-120) en que se narra la lucha entre Glauco y Diomedes, tras la cual los combatientes se dan las manos y se intercambian las respectivas armas. Hay tres grupos de inscripciones en el mosaico, el primero de los cuales en alfabeto griego y los otros dos en latino. En la edición de la Dra.Guardia parece no existir el alfabeto griego y se da a entender, otra vez. que se trata de un solo texto. Por otra parte, aquellas integraciones razonables que procedian de la primera edición de la inscripción (citada en nota 25), fuente reconocida de la Dra.Guardia, no han sido incorporadas a la publicación de 1992.

c(aballus). Un caso paralelo a éste to proporcionaria el mosaico de la cúpula de Centcelles (cf. Schlunk-Hauschild 1978, pags. 119.127 y ( $/ M H$ I, n.T 4). donde encontramos por dos veces $L$. C. En ambos casos podriamos entender la inscripción como un anagrama de propiedad, "caballo de L.... o "caballo de Amorn

"Vid. T.Mañanes-M.A.Gutiérre/-C.Agúndez, El mosaico de la villa romana de Santa Cruz lCabezón de Pisuerga, Valladolid), Valladolid, 1987 y (CIMH I, n.VA I

s. El primero queda comentado supra, s.n. 8.
Fdición Guardia:

$[\ldots]$ TE: MAXECOAI (sic) $\mathrm{h}[\ldots]$
$[\ldots]$ siun $[\ldots]$ eru $[\ldots]$
$[\ldots]$ dif $[\ldots]$
Edición nuestra:

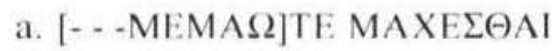

b. HII [-.-]

[T]YDI[DES]
c. [MANV?]S IVN[X]ERVINT]

13. Hay algunas cosas a comentar en las inscripciones del mosaico de Italica en que se representa el nacimiento de Venus ${ }^{17}$. La primera afecta a la unificación tipográfica que debe imperar en la publicación de un grupo de inscripciones. Cuando en la edición de la Dra.Guardia leemos (pág. 408) Ev/poc y en la pág. 405 (cf. el n. 8 de este trabajo) leiamos Pyrra/fili [us] $/$ Tetid/i/s..., tendemos a pensar que la palabra Ey/poc está dividida, en la inscripción, en dos lineas. La realidad del mosaico nos dice que la palabra está dividida por la cabeza del viento

Vid. A. M" Canto, "El mosaico del nacimiento de Venus de ltálicam, Ilabis. 7 (1976), págs 293-338 y CIMH I. n.SE: 5. 

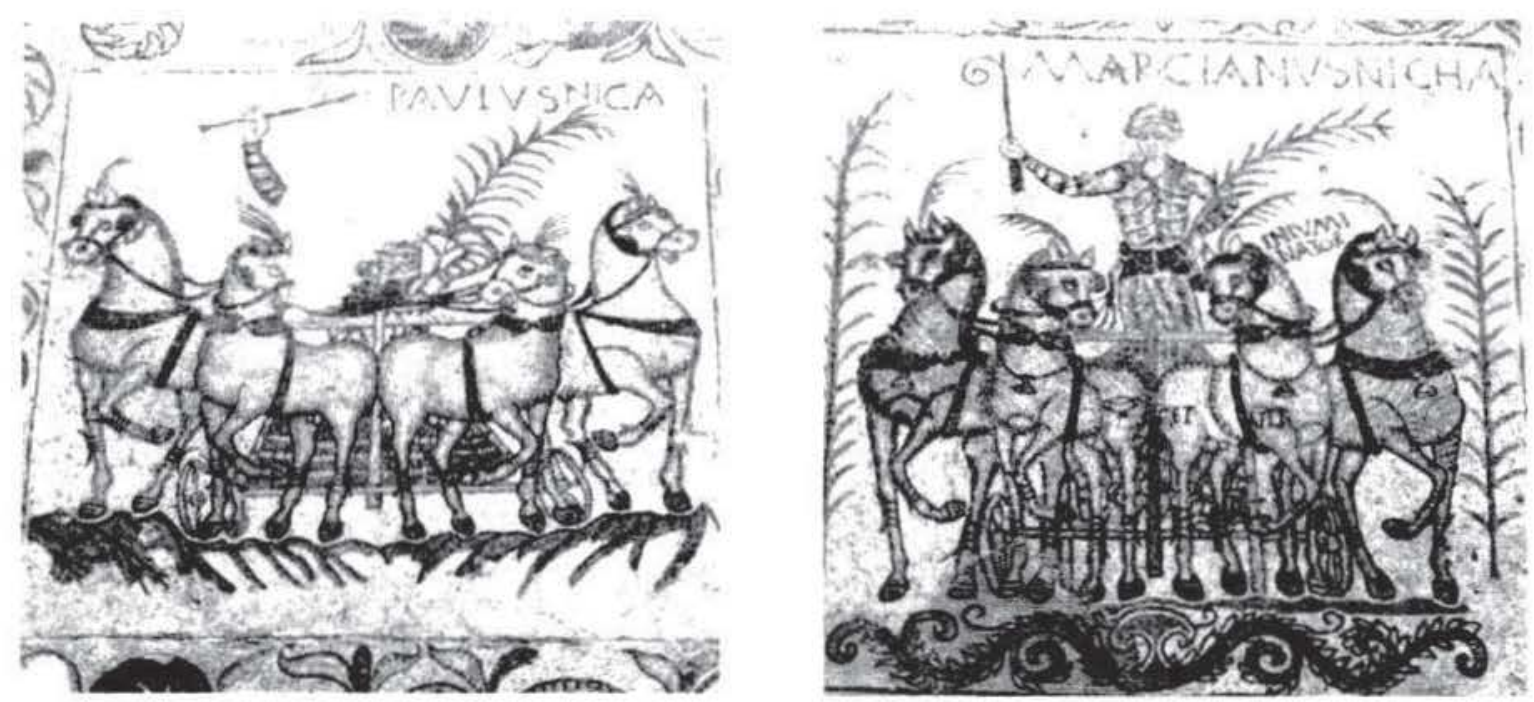

Figura 7. Mosaico de la calle Masona de Merida. Dos cuadrigas con aurigat Victoriosos

que denomina, la primera silaba a su izquierda y la segunda a su derecha.

En segundo lugar. cuando en la pág. 406 (c). n. 10 de este trabajo) encontramos una compaginación como ésta.

\section{Ex oficina Mal-.--|ni \\ Pincit Hirinius \\ Vtere felix Materne \\ Hunc cubiculum}

distinta a las dos anteriores, ya no sabe uno qué pensar sobre la distribución de lineas en las ediciones de la Dra.Guardia en relación con su referente original.

Otro aspecto a comentar es el de la presentación de las inscripciones. Si en este trabajo leemos (edición Guardia)

\section{Ey/poc}

Arethusa

Amimone

¿qué tendemos a pensar? Pues que se trata de un texto único, dividido en tres lineas. ¿O quizá se trata de un texto único dividido en cuatro lineas? Ni lo uno ni lo otro: el emblema central del mosaico está rodeado por octógonos (con figuras de vientos e inscripciones con sus nombres) y rectángulos (la misma configuración, pero con figuras de ninfas). En resumen. la lectura resulta muy dificil porque no hay un único criterio de edición de las inscripciones (en cualquier caso, el de $(\mathrm{ClL} \mathrm{II}$ seguro que no) y la confianza en lo que uno lee se debilita.

Un tercer problema en esta inscripción: como ya es habitual no se indica que existe un nexo en una de las palabras.

Edición nuestra (c\%. figura 6):
a. EY POL
b. ARET^HVSA
c. AMYMONE

14. En un mosaico encontrado en la $\mathrm{c} / \mathrm{Ma}$ sona de Mérida, se representaban dos cuadrigas con aurigae victoriosos ${ }^{2 x}$. Aunque pertenezcan a un mismo pavimento, se trata de dos cuadros compositivos distintos con dos grupos de inscripciones diferenciadas. En la edición de la Dra.Guardia aparecen como si de un solo texto se tratara. Además, falta alguna interpunción; el nombre en genitivo, que probablemente identifica al propietario, se edita Getuli, cuando en el mosaico hay un espacio en blanco

2* Vid. Corpus Mosaicos I, n. 43: Alvare, 1990, pág. 86 y nota 19 y $C I M H I$, n.BA 8. 
partiéndolo ( $G E T V L I$ ). Si la Dra.Guardia hubiera seguido un único criterio (cf. el el/poc del número anterior), aqui se esperaria Get/uli.

\section{Edición Guardia: \\ Paulus Nica \\ Marcianus Nicha \\ Inluminator \\ Getuli}

Edición nuestra (cf. figura 7):

\author{
a. PAVLVS NICA \\ b.I.MAR.CIANVS . NICHA \\ b.2. INLVMI \\ NATOR \\ b.3. GET VLI
}

15. En la presentación del mosaico de Anniponus ${ }^{20}$, se tendría que corregir «mosaico de Annibonu» por "mosaico de Annibonus».

16. En las inscripciones que proceden de Santa Vitoria do Ameixial (Estremoz, Evora) vemos dos problemas.

El primero afecta a la utilización de la minúscula y la mayúscula en las ediciones de la Dra.Guardia. Vemos cómo la única inscripción griega de la villa portuguesa ${ }^{30}$ se edita en mayúsculas, mientras que las demás vienen editadas en minúsculas, sin que sepamos a qué criterio obedece el cambio.

En esta primera inscripción, griega ${ }^{31}$, no se indica en ningún momento que existe un espacio perdido en la parte izquierda del mosaico.

\section{Edición Guardia:}

\section{IONYCI /TWHP / K $\lambda \mathrm{HMH} / \mathrm{O} \lambda \mathrm{IKEN}$}

29 No quiero entrar en estas páginas en la discusión sobre la lectura de este nombre: hay especialistas que leen Anni Boni, otros Anniboni (como la Dra.Guardia, p.410). algunos más Anni Poni y unos pocos, entre los cuales yo. Anniponi, Remitimos a Donderer 1989, n.A 47, Gómez Pallarès 1991, n. 1.12 y CIMH I, n.BA 9.

" Por primera vez encontramos una «lambda» griega en la tipografía de este libro. El problema está en que el texto está editado en mayúsculas y la «lambda", en minúsculas ( $\lambda$ en vez de $\Lambda$ ). Por otra parte. lo que debia de ser una "ómega" mayúscula $(\Omega)$, se convierte en una "wau» indo-europea $(W)$.

3 Vid. Chaves 1956, pág. 57; Fernández-Galiano 1984. pág. 125; Alarcâo 1988, págs. 154-155 y CIMH I, n.POR 6.
Edición nuestra:

$$
\begin{aligned}
& {[--] \text { IONYSI }} \\
& {[--] \text { TSHP }} \\
& {[\cdots] \text { KAHMH }} \\
& [-\cdots] \Omega \text { - }] \text { SIKEN }
\end{aligned}
$$

El segundo problema hace referencia a la corrección de las pruebas de imprenta. Todos conocemos el peligro de una edición epigráfica y la minuciosidad con que deben ser corregidos los trabajos. Algunos de estos problemas han sido ya indicados supra, pero el más grave de ellos llega cuando falta una parte del texto de una inscripción. Esto ocurre con la inscripción musiva de la llamada Sala $C$ de Santa Vitoria do Ameixial, en la que encontramos imágenes de vientos con sus letreros identificativos, rodeando el emblema central ${ }^{32}$. Faltan dos de los nombres de vientos.

\section{Edición Guardia: \\ Eurus Notus \\ Edición nuestra:}
a. EVRVS (hedera)
b. ZEPH[YRVS]
c. BOR[EAS]
d. NOTVS

17. En Puebla de la Calzada (y no Valdelacalzada, como indica la Dra.Guardia en la pág. 412), se excavó una villa, en la que apareció un pavimento con motivos geométricos y firma de taller musivario ${ }^{33}$. La Dra.Guardia se equivoca en la lectura y en la compaginación del texto.

\section{Edición Guardia:}

Ex officina Dexter (sic)

Edición nuestra:

\section{EX OFFICINA DEXTERI}

12 Vid. Chaves 1956, pảgs, 44-46; Alarcâo 1988, págs. 154-155 y CIMH I, n.POR 5.

"Vid. Lancha 1984, p.51; Donderer 1989, n.A 55 HE I 116: Gómez Pallarès 1991, n.1.13 y CIMH I. n. BA 10. 
18. ¿Conclusiones? Un trabajo que nace de la recopilación de algunas observaciones a un fragmento de libro no necesita de conclusiones stricto sensu. En mi opinión habria sido mucho mejor, si la voluntad de la Dra.Guardia era publicar los apéndices con las inscripciones de los pavimentos musivos estudiados por ella, que hubiera realizado una lectura más crítica y rigurosa de esas inscripciones. Su trabajo en el campo de la iconografía es realmente importante y no hacia ninguna falta enturbiarlo con una información imprecisa y no pocas veces incierta. Si sus ediciones hubieran sido fiables, su trabajo global hubiera resultado doblemente valioso, porque hubiéramos encontrado en un solo volumen una correcta edición de textos y un mejor comentario de las imágenes a que acompañan, y esa es, a no dudarlo, la mejor manera de comprender una inscripción musiva.
No me complace especialmente escribir páginas como éstas, pero pienso que es mucho mejor corregir los errores de lectura publicados por la Dra.Guardia que no pensar que los lectores no especializados en el tema van a recibir una información incorrecta. Tómense estas páginas como un complemento al dossier de los mosaicos bajo-imperiales en Hispania ${ }^{34}$.

\footnotetext{
4 Créditos de las fotografias:

figura I: foto de IRC II, pl.VIII.

figura 2: foto de J.Gómez Pallarès

figura 3: foto de J.Gómez Pallarès

figura 4: foto de Foto Estudio Tria, Jaén.

figura 5: foto de Donderer 1989. Taf.40.2

figura 6: fotos del Área de Difusión del Conjunto Arqueológico de Itálica.

figura 7: fotos de J.Gómez Pallarès.
}

\title{
LATE ROMAN UNGUENTARIUM: UNGÜENTARIOS CRISTIANOS DE LA ANTIGÜEDAD TARDÍA PROCEDENTES DE PUNTA DE L'ILLA DE CULLERA, VALENCIA'
}

\author{
POR \\ M. ${ }^{\mathrm{a}}$ ISABEL GARCIA VILLANUEVA \\ MIQUEL ROSSELLÓ MESQUIDA \\ Servicio de Investigación Arqueológica Municipal de Valencia (S.I.A.M.)
}

RESUMEN

Presentamos en este articulo un conjunto de ungüentarios cerámicos de origen oriental de la Antigüedad Tardia procedentes de Punta de I'Illa de Cullera, Valencia. El interés de estas piezas viene dado por ser la primera vez que se documentan en la Península y su posible relación con algún aspecto de la liturgia cristiana.

' Queremos agradecer al Servicio de Investigación Prehistórica de ta Diputación de Valencia y especialmente a su Director, Dr. Bernat Marti Oliver, las facilidades y el
SUMMARY

In this paper we want to present a type of pottery production (Late Roman Unguentarium) characteristic of the Eastern Mediterranean area from the sixth and seventh century A.D., found at "Punta de I'Illa de Culleran site, in Valencia.

interés mostrado en la realización de este trabajo. Asi mismo, agradecemos al Dr. Luis Caballero Zoreda y a Dña. Rafaela Soriano Sánchez la preocupación e interés en la elaboración de este artículo. 